\title{
Hormonal Pathology of the Endometrium
}

Liane Deligdisch, M.D.

Departments of Pathology and Obstetrics-Gynecology and Reproductive Science, The Mount Sinai-NYU

Medical Center, New York, New York

The endometrial tissue is a sensitive target for steroid sex hormones and is able to modify its structural characteristics with promptness and versatility. This article discusses briefly endogenous hormonal effects (cyclic changes, luteal phase defect, unopposed estrogen effect) and describes the histologic patterns encountered in the most commonly used hormone therapies: oral contraceptives, ovulation stimulation, hormone replacement therapy, and antitumoral hormone therapy.

Oral contraceptives exert a predominant progestational effect on the endometriun, inducing an arrest of glandular proliferation, pseudosecretion, and stromal edema followed by decidualized stroma with granulocytes and thin sinusoidal blood vessels. Prolonged use results in progressive endometrial atrophy.

Ovulation induction therapy accelerates the maturation of the stroma and is often associated with a discrepancy between early secretory glands and an edematous or decidualized stroma with spiral arterioles.

Hormone replacement therapy with estrogen alone may result in continuous endometrial proliferation, hyperplasia, and neoplasia. The use of both estrogen and progesterone elicits a wide range of histologic patterns, seen in various combinations: proliferative and secretory changes, often mixed in the same tissue sample; glandular hyperplasia (in polyps or diffuse) ranging from simple to complex atypical; stromal hyperplasia and/or decidual transformation; epithelial metaplasia (eosinophilic, ciliated, mucinous); and inactive and atrophic endometrium.

Progesterone therapy for endometrial hyperplasia and neoplasia induces glandular secretory changes, decidual reaction, and spiral arterioles. Glandular proliferation is usually arrested, but neo-

Copyright (C) 2000 by The United States and Canadian Academy of Pathology, Inc.

VOL. 13, NO. 3, P. 285, 2000 Printed in the U.S.A.

Date of acceptance: November 8, 1999.

Address reprint requests to: Liane Deligdisch, M.D., Mount Sinai Medical

Center, Department of Pathology, One Gustave Levy Place, New York, NY 10029.

plastic changes may persist and coexist with secretory changes.

Lupron therapy produces a shrinking of uterine leiomyomas by accelerating their hyaline degeneration, similar to that in postmenopausal involution. It generally produces endometrial atrophy.

Tamoxifen for breast carcinoma has an estrogenagonist effect on the uterus in approximately $20 \%$ of patients, who develop endometrial polyps, glandular hyperplasia, adenomyosis, and/or leiomyomata. Both endometrioid and nonendometrioid carcinomas are seen, often in polyps. Their causal relationship to tamoxifen therapy is debatable.

Mod Pathol 2000;13(3):285-294

Hormone therapy is widely used throughout the world by women of all ages for a variety of reasons, ranging from oral contraception and ovulation stimulation for family planning to hormone replacement therapy in menopause, to adjuvant therapy of tumors of the breast and uterus. The histopathologic changes of the uterus, and particularly of the endometrium, associated with these therapies encompass a variety of morphologic features that are often difficult to interpret. The endometrium is a sensitive target tissue for steroid sex hormones and is able to modify its structural characteristics with promptness and versatility. The physiologic changes of the endometrium during reproductive life and after menopause reflect the influence of ovarian-secreted steroid sex hormones and of their withdrawal. Hormone manipulation in fertility problems and hormonal substitution therapy result in histologic patterns that do not fit the classical descriptions of the cyclic and involutional changes of the endometrium. Various combinations of hyperplastic, proliferative, secretory, and atrophic changes of endometrial glands, stroma, and blood vessels may result in confusing histologic patterns. As hormone therapy changes over time, with new regimens continuously being implemented, the effect on the endometrium can result in unpredictable structural changes.

The histologic patterns of the endometrium associated with hormone therapy vary with the dosage, duration of therapy, and individual hormone receptor activity. The same endometrium may dis- 
play a number of changes under different conditions, resulting in a diversity of histologic patterns that may render some endometrial biopsies difficult to interpret. Although the endometrial response may vary from patient to patient, certain general histologic patterns for specific hormone therapies can be recognized. To comprehend the iatrogenic effect of exogenous hormone therapy, a review of the normal and abnormal endogenous hormone-related endometrial changes is appropriate.

\section{ENDOMETRIAL MORPHOLOGY AND PATHOPHYSIOLOGY}

Before puberty, the endometrial tissue is inactive; it is composed of tubular glands, a dense fibroblastic stroma, and thin blood vessels. The advent of cyclic pituitary and ovarian hormonal activity results in endometrial cyclic morphologic changes involving glands, stroma, and blood vessels that can be identified as characteristic for each day of the cycle.

In normal cycles, the menstrual shedding is followed by endometrial proliferation under estrogenic stimulation. The endometrial thickness increases more than 10 -fold as a result of active growth of glands, stroma, and blood vessels. The proliferative phase has a variable length from 10 to 20 days, with an ideal duration of 14 days. During this phase, the endometrial glands grow and become tortuous because of the active proliferation of epithelial cells. These estrogen-induced changes occur as a response to the binding of the hormone to nuclear receptors. The presence of estrogen receptors in the nuclei of endometrial cells is responsible for the prompt translation of hormonal impulses into structural changes, including, at an ultrastructural level, protein synthesis by free ribosomes and by the rough endoplasmic reticulum and accumulation of intermediate filaments, mitochondria, Golgi apparatus, and lysosomes. The nuclei increase in size, nucleoli become prominent, and chromatin is coarse. The individual cell mass increases as does the nuclear-cytoplasmic ratio. Numerous mitoses are seen throughout the glands and stroma.

After ovulation, the secretion of progesterone inhibits the proliferative activity of the endometrium and induces a complex secretory activity starting with the polarization of glycogen at a subnuclear location followed by its transport via microfilaments to the apical region of the cell. The Golgi apparatus "packages" the glycogen and various other substances, which become secretory granules and are eventually expelled into the glandular lumen. The secretory changes take place only in an estrogen-primed endometrium.
The secretory activity in the second half of the menstrual cycle is characterized by a diversity of structural changes that are apparent on routine examination of endometrial biopsies, showing a different pattern on every day of the cycle. "Dating" the endometrium is identifying morphologic changes characteristic for early, middle, and late proliferative endometrium and for each of the 14 days of secretory endometrium $(1,2)$.

Perhaps the most significant change in terms of adequacy of the luteal phase is that involving the blood vessels. The thin endometrial arterioles undergo a process of endothelial proliferation, thickening of the wall, and coiling forming the spiral arterioles on the ninth postovulatory day. These arterioles have a critical role in the process of implantation because of the tropism for arterial blood that characterizes trophoblastic tissue. Their coiling increases the surface to be "tapped" by the implanting trophoblast; therefore, the chances for a normal implantation are reduced if the spiral arterioles are not well developed. The evaluation of endometrial biopsies properly performed a few days before the onset of menstrual shedding is one of the most important diagnostic tools in the work-up of infertility: It offers an insight into the tissue that will play host to the implanting conceptus, assessing its adequacy.

Pathologists are often asked to evaluate luteal phase defects (LPD) in their reports on endometrial biopsies. LPD considered to be due to an inadequate progesterone production is clinically sometimes associated with infertility and with spontaneous abortions. Histologically, the maturation of the endometrium is most commonly associated with a lag of more than 2 days of the histologic date from the actual postovulatory date, possibly the result of delayed ovulation (3). In insufficient follicle maturation, the endometrium appears immature; in persistent graafian follicle, proliferative changes persist throughout the secretory phase. A dissociation between glandular and stromal maturation can also be seen in LPD (see Table 1). The pathogenesis of LPD is not completely understood. It may occur when the corpus luteum fails to develop adequately or as a result of deficient progesterone receptors. To be diagnostically significant, LPD has to be found in at least two consecutive cycles (3).

TABLE 1. Luteal Phase Defect

\begin{tabular}{lc}
\hline \multicolumn{1}{c}{ Ovarian Changes } & Endometrial Changes \\
\hline $\begin{array}{l}\text { Delayed ovulation } \\
\text { Insufficient follicle maturation }\end{array}$ & $\begin{array}{c}\text { Thin endometrium, low glycogen, thin } \\
\text { arterioles, tubular glands }\end{array}$ \\
$\begin{array}{l}\text { Persistent graafian follicle- } \\
\text { high estrogen secretion }\end{array}$ & $\begin{array}{c}\text { Mitotic activity in secretory glands, } \\
\text { stratified epithelium (mixed } \\
\text { endometrium) }\end{array}$ \\
$?$ & Dissociated gland-stroma maturation \\
\hline
\end{tabular}


After menopause, the endometrium normally undergoes a gradual atrophy, starting with an inactive phase in which neither proliferation nor secretion is present and ending up as a thin layer, often riddled with cystic cavities lined by a cuboidal or flat epithelium, in which the organelles are pushed to random locations and the stroma becomes fibrotic (4).

Failure to ovulate, after menopause, first deprives the endometrium of progesteronic stimulation. The estrogenic stimulation, however, may continue because of the conversion of androgens, secreted by the menopausal ovaries and adrenal cortices, into estrogens. Obesity, diabetes, and other metabolic disorders may enhance the extragonadal endogenous estrogen production by aromatization. High estrogen levels, especially of estradiol, are often associated with endometrial hyperplasia because of the binding of the hormone to receptor sites in the nuclei of endometrial cells (5).

The response of the endometrial tissue to a continuous, prolonged, and unopposed (by progesterone) estrogenic stimulation in postmenopausal women can be continuous proliferation, hyperplasia, and, possibly, neoplasia. Endometrial hyperplasia and neoplasia are less common in younger women, where they are associated with anovulatory cycles, such as is the case, for example, in polycystic ovarian disease (PCOD).

\section{IATROGENIC HORMONAL EFFECTS}

\section{Oral Contraceptives}

It is estimated that more than 50 million women in the world, including at least 10 million in the United States, use oral contraceptives (OC), an effective and accessible method of contraception. Most OC contain small dosages of estrogens (ethinyl estradiol) and of testosterone-derived progesterone (norethindrone), whereas some contain only progestins (Micronor, Ovrette).

The mechanism of preventing pregnancy is based on the notion that the presence of one pregnancy prevents the onset of another, therefore the administration of hormonal agents secreted during pregnancy (estrogen and progesterone) would suppress ovulation and implantation.

Historically, OC were used in sequential or combined fashion. The sequential OC were meant to mimic the natural cycle and consisted of administration of estrogens during the first 5 to 19 days of the cycle followed by the addition of progestins until day 24, after which withdrawal of the hormones produced breakthrough bleeding. This regimen was neither completely safe nor completely effective: Cases of endometrial hyperplasia and even neoplasia were reported (presumably because of the use of estrogens alone) as well as unwanted pregnancies $(6,7)$. Sequential OC therapy was discontinued in the United States in 1976.

The dosage and potency of the hormones used for OC were much higher at the inception of OC usage and were associated with a relative high incidence of undesirable side effects, such as thrombosis and migraine. Presently, combination OC containing lower dosages of hormones are used with high efficiency and fewer side effects.

The effect of OC on the endometrium is related to the dosage and potency of the hormones. The progesterone effect dominates over the estrogen effect. High-dose, high-potency OC produce endometrial stromal cell hyperplasia, decidual reaction, and glandular atrophy. The stromal hyperplasia may be atypical, suggestive of stromal neoplasms (8). Blood vessels can display endothelial hyperplasia and marked thickening of the wall as well as smooth muscle hyperplasia. High-dose, high-potency OC are not used any more as contraceptives but are occasionally administered to patients with endometrial hyperplasia, resulting in marked endometrial stromal cell hyperplasia (Fig. 1).

The OC currently in use, containing small dosages of estrogen and progesterone, produce an ar-

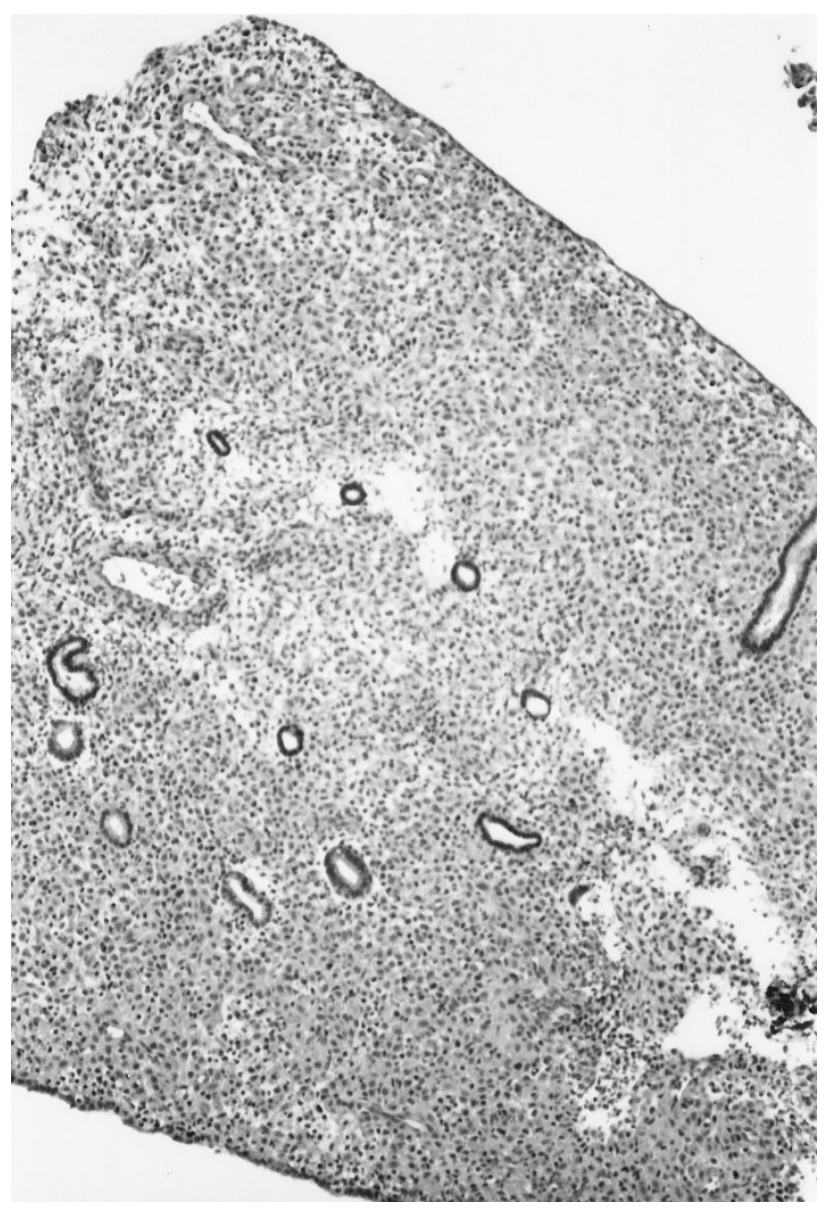

FIGURE 1. High-potency progesterone oral contraceptive (used for endometrial hyperplasia) effect: small inactive glands, hyperplastic stroma, and thickened blood vessels. 
rest of endometrial glandular proliferation during the first cycles. The glands are straight or slightly coiled and are lined by immature epithelial cells with nuclei showing an evenly distributed chromatin network. The thick nuclear membrane, coarsely clumped chromatin, and mitotic activity seen in proliferative endometrium are absent. The cytoplasm contains randomly distributed vacuoles, and the apical border, unlike that in secretory endometrium, is smooth and well defined. The glands may contain some eosinophilic dense secretion ("abortive" secretion). The glands are unevenly distributed, with abundant stroma among them. Early in OC therapy, there is stromal edema, followed later by a decidual reaction and granulocytic infiltrates. The blood vessels do not undergo the changes characteristic for spiral arterioles, which are seen in normal cycles with decidualized stroma. They become thinner, often dilated like sinusoids, after prolonged therapy (Fig. 2). In breakthrough bleeding, the veins may become sinusoidal and thrombosed. Prolonged use of OC results in the disappearance of the abortive glandular secretion and in atrophy of both glands and stroma; occasionally, an abundant stroma and ectatic blood vessels can be found. On ultrastructure, there is a progressive disappearance of cilia and nucleolar channel system (9). Also seen in women who take OC is endocervical gland hyperplasia, most often microglandular hyperplasia with squamous metaplasia, similar to that seen during pregnancy.

The histologic patterns seen in endometrial biopsies from women who receive OC therapy do not resemble any "natural" noniatrogenic endometrial change, normal or pathologic. The combination of inactive glands, abortive secretion, decidual reaction, and thin blood vessels is characteristic for OC therapy effect (Table 2).

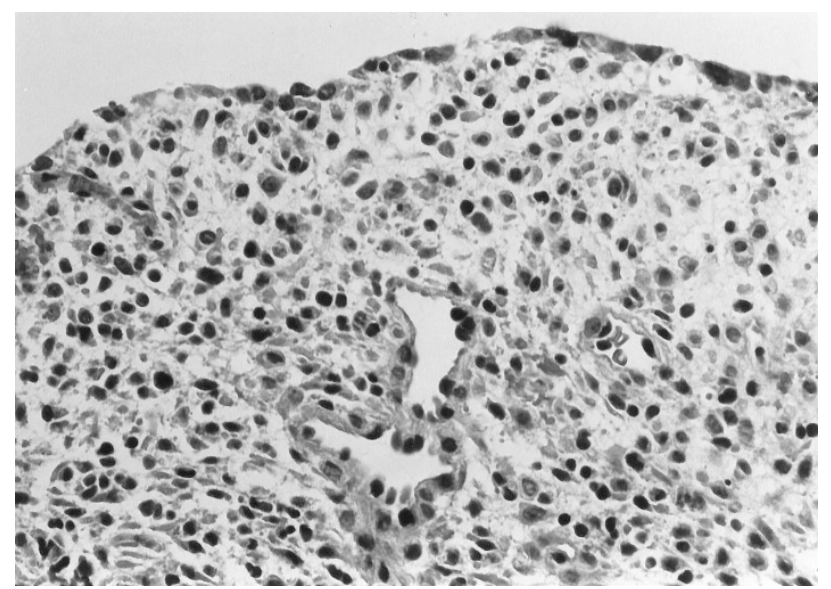

FIGURE 2. Commonly used oral contraceptive effect: atrophic endometrial epithelium, stromal decidual reaction, and thin dilated blood vessels.
TABLE 2. Endometrial Histology in Oral Contraceptive Therapy

\begin{tabular}{ll}
\hline \multicolumn{1}{c}{ Commonly Used } & \multicolumn{1}{c}{ High Potency } \\
\hline Arrest of glandular proliferation & Glandular atrophy \\
Abortive secretion & Stromal hyperplasia \\
Low gland/stroma proportion & Pseudosarcoma \\
Stromal edema & Vascular thickening \\
Decidua, granulocytes & Stromal leiomyomas \\
Thin blood vessels, venous sinusoids & Decidua \\
Atrophy & \\
\hline
\end{tabular}

\section{Ovulation Induction Therapy}

Ovulation failure is the primary defect in at least $20 \%$ of infertile couples. It is manifested as irregular and/or infrequent ovulation or chronic anovulation as a result of deficient gonadotropins and their inability to stimulate follicle maturation.

Induction of ovulation by hyperstimulation of the ovaries results in the selection of multiple oocytes in one cycle. The ova can be retrieved and used for in vitro fertilization. Ovulation induction therapy is directed at correcting the primary dysfunction causing anovulation by producing augmentation of endogenous gonadotropin. The most commonly used agents are clomiphene citrate, human menopausal gonadotropins, gonadotropin releasing hormone, and human chorionic gonadotropins. Various combinations of these drugs are used in the treatment of infertility (10). Clomiphene citrate has been used successfully for anovulatory cycles and to correct LPD; however, it was shown that it may alter steroid receptor kinetics by competitively lowering the estrogen receptors in the endometrium. Inhibition of progesterone receptors has been thought to be responsible for LPD (11).

An optimal follicular development with adequate estrogen secretion is crucial for subsequent endometrial maturation. With ovulation induction therapy, the endometrial maturation process is enhanced by accelerating the secretory changes in the stroma $(12,13)$. A disparity between more advanced stromal maturation and less advanced glandular maturation is often found in endometrial biopsies from patients treated with ovulation induction. The glands display early secretory features, mostly subnuclear vacuoles, consistent with days 16 to 17 (or second to third postovulatory day in normal cycles), whereas the stromal changes include edema and spiral arterioles, corresponding to days 22 to 23 (8 to 9 postovulation) (Fig. 3). Progesterone therapy to correct this disparity is often successful.

In rare cases, especially in patients whose anovulation was associated with PCOD, persistent endometrial hyperplasia is seen along with secretory changes of the glands and stroma. In a small number of such cases, there is atypical glandular hyperplasia with cribriforming and even overt neoplasia. Endometrial carcinoma has been identified in abor- 


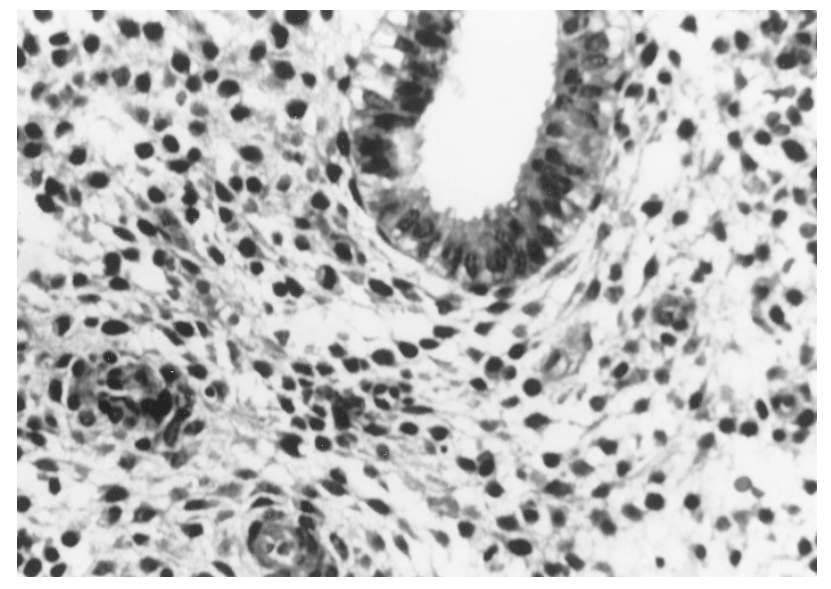

FIGURE 3. Ovulation induction therapy: endometrial glands with early secretory changes with subnuclear vacuoles, consistent with days 16 to 17, and stroma with edema and spiral arterioles, consistent with days 22 to 23 .

tion material from a patient treated with ovulation induction for PCOD (Fig. 4).

\section{Hormone Replacement Therapy}

With the prolongation of life expectancy, the postmenopausal period may last up to one third or more of a woman's lifetime. A large number of women are presently treated with hormone replacement therapy (HRT) for the prevention of postmenopausal morbidity as a result of the progressive decrease of natural estrogen secretion. Hot flashes, urogenital atrophy, insomnia, and anxiety are among the most common symptoms. Lipid metabolism disorders may lead to a higher, lifethreatening morbidity as a result of cardiovascular diseases and osteoporosis.

Estrogens and estrogen derivatives were used for a few decades. It became clear in the 1970s that the administration of estrogen alone, used for the pre-

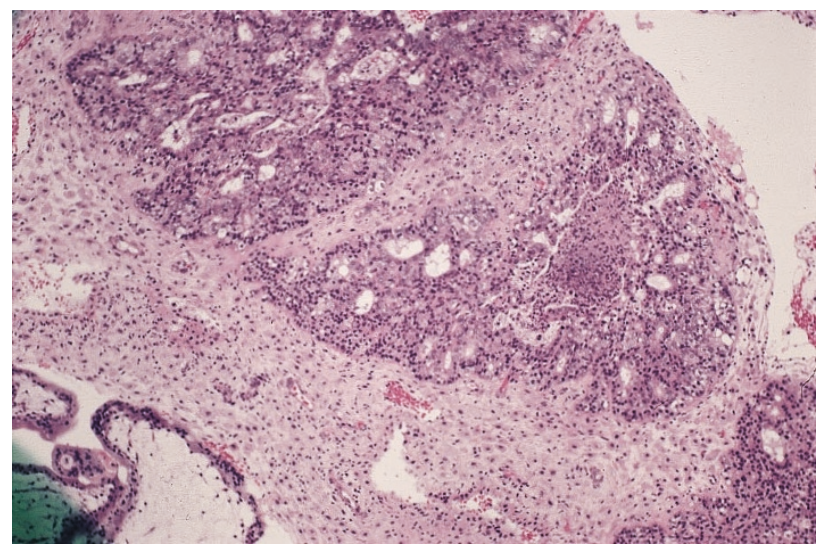

FIGURE 4. Ovulation induction therapy for polycystic ovarian disease with endometrial complex atypical hyperplasia and infertility. The patient conceived and had a spontaneous abortion. Endometrial curettings showed chorionic villi (left lower corner) and focal welldifferentiated adenocarcinoma. vious 2 to 3 decades, may be associated with endometrial hyperplasia and neoplasia (14-16). Since then, combined and sequential regimens of HRT have been studied extensively and administered on a large scale. The estrogen/progesterone HRT regimens are different from those used for oral contraception, mainly because of the significantly lower dosages and the different estrogens used. The commonly used estrogen preparations are conjugated equine estrogen and natural estradiol in micronized form, and the most commonly used progesterone is medroxyprogesterone acetate (MPA). Combined estrogen/progesterone HRT is widely prescribed to millions of women (17-19). It is used in either cyclic or continuous fashion. The most commonly used cyclic regimen consists of conjugated estrogens (Premarin $0.625 \mathrm{mg}$ ) to which MPA (Provera) 2.5 or $5 \mathrm{mg}$ is added for the last 10 to 14 days of estrogen therapy. In continuous HRT, the latter is given at 2.5 or $5 \mathrm{mg}$ daily. More recent studies propose HRT with even lower doses. HRT is also prescribed to younger women who have been diagnosed with premature ovarian failure or Turner's syndrome or who have undergone prophylactic oophorectomy.

Despite the fact that the risk of estrogen therapy alone in promoting endometrial and breast neoplasms became clear over the past 3 to 4 decades, there are postmenopausal women who do not tolerate some side effects of progesterone and choose to be treated by estrogen only.

The endometrial changes associated with unopposed estrogen therapy are positively related to the duration of the exposure. They consist of proliferative endometrium (sometimes weakly proliferative), often with stromal breakdown, and/or of endometrial hyperplasia ranging from simple to complex and atypical, with occasional presence of squamoid metaplasia ("morules") (Fig. 5) and stromal foam

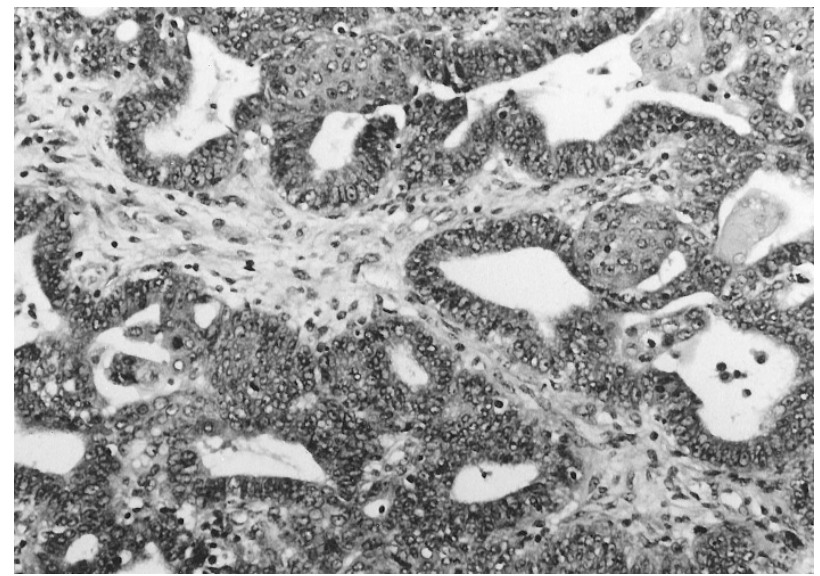

FIGURE 5. Hormone replacement therapy: 56-year-old postmenopausal woman treated for 4 years with estrogen alone. Endometrial complex glandular hyperplasia with squamoid morules. 
cells. These changes may persist after the cessation of therapy (Fig. 6). Endometrial polyps with hyperplastic changes ranging from simple to atypical can also be seen. Endometrial carcinoma, usually but not necessarily always of well-differentiated endometrioid type, is encountered more often in patients treated with estrogens alone for a longer period of time $(15,16)$.

The effect of combined HRT on the endometrium includes a wide spectrum of morphologic features $(18,20)$. Various combinations of weakly to intensely proliferative glands and/or secretory glands, with sub- and supranuclear vacuoles and luminal secretion, often surrounded by stromal edema, stromal hyperplasia, and decidual reaction, are seen. This "mixed" proliferative and secretory endometrium may include crowded and hyperplastic glands (Fig. 7) alternating with an edematous, hyperplastic, or decidualized stroma, sometimes with thickened or thrombosed blood vessels. The resulting histologic pattern may appear rather confusing, especially if referred to the natural cyclic endometrial dating system. Associated features can include tubal, eosinophilic, mucinous, or papillary metaplasia presenting an irregular pattern that, especially when combined with hyperplastic changes, may appear suspicious for neoplasm. It is not unusual to see some of these patterns along with menstrual changes (breakdown of stroma, fibrinoid intravascular thrombi, polymorphonuclear leukocytic infiltrates, cellular debris with nuclear "dust"), probably as a result of the iatrogenic withdrawal of hormones (Table 3). The most commonly observed histologic pattern associated with the currently widely used Premarin-Provera (Prempro) regimen is that of proliferative glands and moderately hyperplastic stroma.

Inactive and atrophic endometrium is often encountered in endometrial biopsies from women who are receiving HRT, occasionally associated

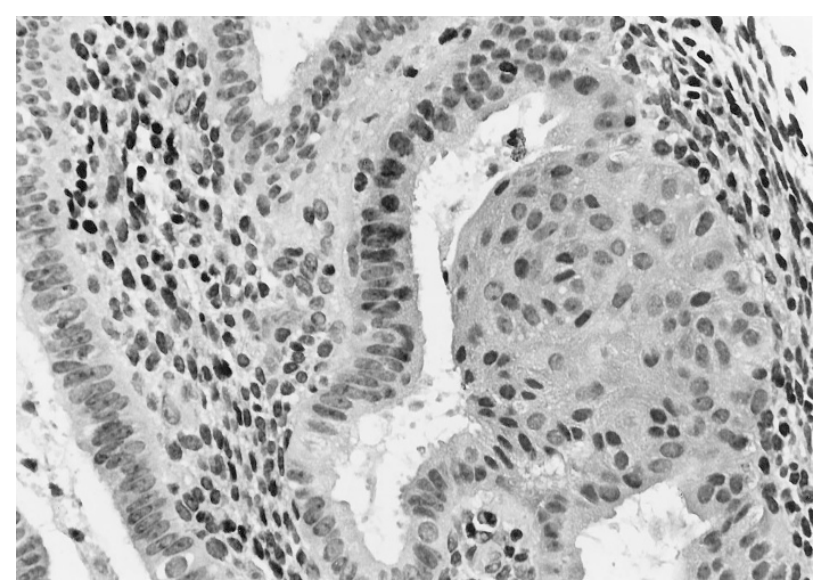

FIGURE 6. Same patient as Figure 5 after 6 months of combined estrogen-progesterone therapy. The glands appear quiescent and focally secretory; squamoid morules persist.

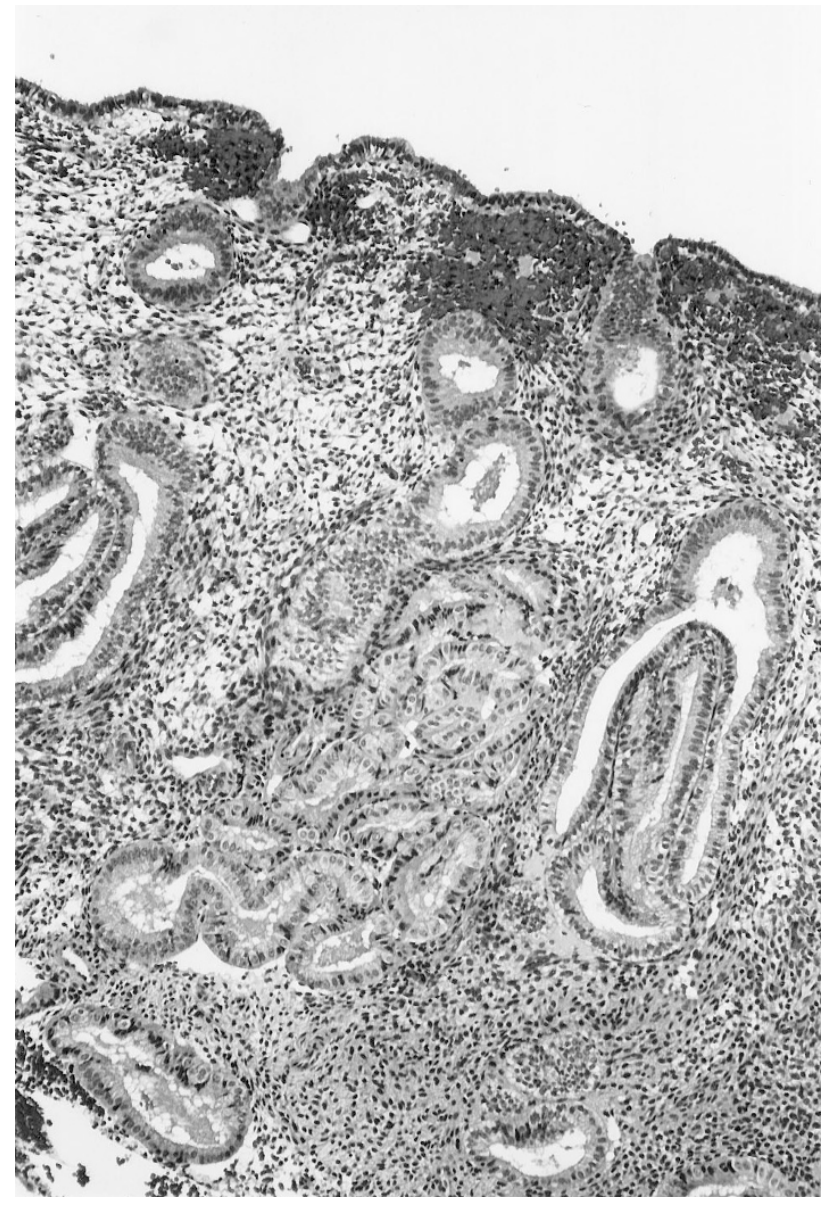

FIGURE 7. Hormone replacement therapy of postmenopausal 59year-old woman with combined estrogen/progesterone (Prempro).

Mixed endometrium: secretory endometrium with focally hyperplastic glands.

\section{TABLE 3. Hormone Replacement Therapy}

Sequential estrogen-gestagen in 25-day cycle

Continuous estrogen, gestagen supplement 10 to 12 days per month

Continuous combined estrogen/progesterone

Effects on endometrial histology

Proliferation-secretion-mixed-menstrual

Glandular hyperplasia: simple, complex, atypical

Metaplasia: tubal, mucinous, papillary

Stromal edema, decidua, spiral arterioles

Weakly proliferative, secretory, inactive, atrophic

Any combination of the above

with functional and/or hyperplastic changes. In prolonged HRT, the endometrium should become gradually atrophic as a result of the exhaustion of receptors. The extent of atrophic, functional, and hyperplastic changes, however, cannot be assessed because most asymptomatic women who are receiving HRT do not undergo endometrial biopsies. Often seen in women who are receiving combined hormone therapy is endocervical glandular hyperplasia.

It is presumed that neoplastic changes are prevented by adding progesterone to estrogen therapy; this is, however, not always the case; occasionally, 
endometrial carcinoma occurs in women who have been treated with combined HRT (Fig. 8) $(21,22)$. Recent studies confirmed the lower risk of endometrial cancer associated with use of combined estrogen/progestagen regimens than with unopposed estrogen alone but stressed the higher risk of endometrial cancer in women who are receiving cyclic progesterone with a shorter duration of the progestagen administration (23).

\section{Antineoplastic Hormone Therapy}

\section{For uterine neoplasms}

Endometrial hyperplasia and neoplasia are often treated with progesterone, especially in younger women who want to have children. The progesterone therapy used for endometrial hyperplasia without atypia is MPA 10 to $20 \mathrm{mg}$ daily, 14 days per month, or continuously 20 to $40 \mathrm{mg}$ daily. MPA is also given as an adjuvant therapy for endometrial carcinoma. High-dose progestins are prescribed to young women who want to have children and to selected postmenopausal patients with contraindications for surgery, for three to six cycles. The progesterone therapy for endometrial hyperplasia and neoplasia is based on its ability to inhibit DNA synthesis and to induce regression of abnormal endometrial proliferation (24-27).

The histologic response to progesterone therapy is mediated by progesterone receptors. The intensity of the response correlates with the receptor levels $(28,29)$. This therapy produces an arrest of glandular proliferation, with secretory changes in the glands and decidual reaction in the stroma. The endometrial tissue appears quiescent, with no mitotic activity. Secretory changes, however, may alternate with hyperplastic glands.

Consecutive endometrial biopsies of patients diagnosed as having endometrial hyperplasia and treated with progesterone may show different patterns, with a progressive decrease of the hyperplastic changes, in cases of simple and complex hyperplasia. Secretory changes such as subnuclear vacuoles, luminal secretion, stromal hyperplasia with decidual reaction, and thickened, coiled arterioles may be seen in various combinations. Cystic glands often persist, as well as occasional glandular crowding. In atypical glandular hyperplasia and in adenocarcinoma, adjuvant progesterone therapy may fail to produce any effect or may produce only partial changes: Parts of the endometrium show secretory changes in glands that remain crowded and irregular in shape (Fig. 9). Cribriform glandular patterns encountered in endometrial neoplasms are not likely to be influenced by progesterone therapy even though the epithelial cells may show diminished or absent mitotic activity and the presence of cytoplasmic secretory vacuoles (Fig. 10). Generally, the architecture of the endometrial tissue shows less change after progesterone therapy than do the individual cells. Cystic glands, crowding, outpouching, and irregularly shaped glands may persist, while the epithelial cells become low columnar or cuboidal, with round nuclei containing a delicate network of chromatin, secretory vacuoles in the cytoplasm, and little or no mitotic activity. Squamoid metaplasia (morules) may persist after therapy as well. Sometimes, an irregular sawtooth pattern characteristic of late secretory change may mimic neoplastic glandular crowding, which is more irregular and lined by more active-appearing epithelial cells.

It is important to be aware that the finding of progesterone-induced changes, including decidual transformation, in an endometrial biopsy after progesterone therapy for atypical hyperplasia or adenocarcinoma does not necessarily mean that the neoplastic change has completely reverted. Only at

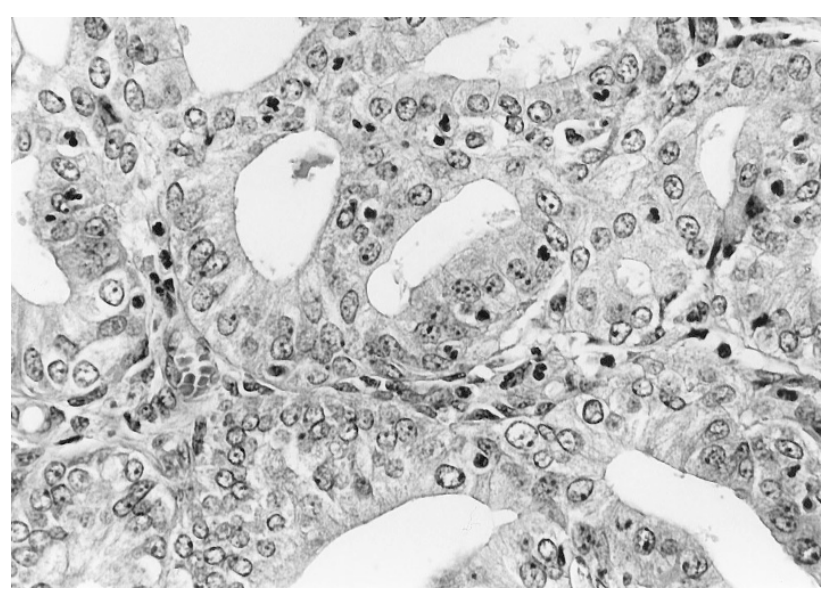

FIGURE 8. Hormone replacement therapy for 10 years with estrogen and cyclic progesterone added for 10 days a month in a 71-year-old patient: well-differentiated adenocarcinoma.

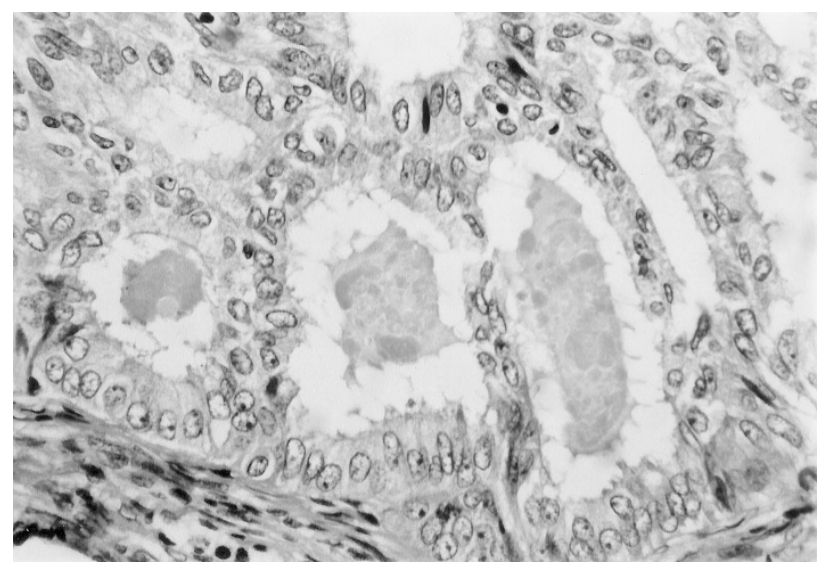

FIGURE 9. Progesterone therapy for endometrial neoplasm: 32-yearold patient with endometrial carcinoma, after 6 months of therapy with medroxyprogesterone acetate $40 \mathrm{mg}$ daily. Endometrial biopsy shows back-to-back crowded glands with intraluminal secretion. 


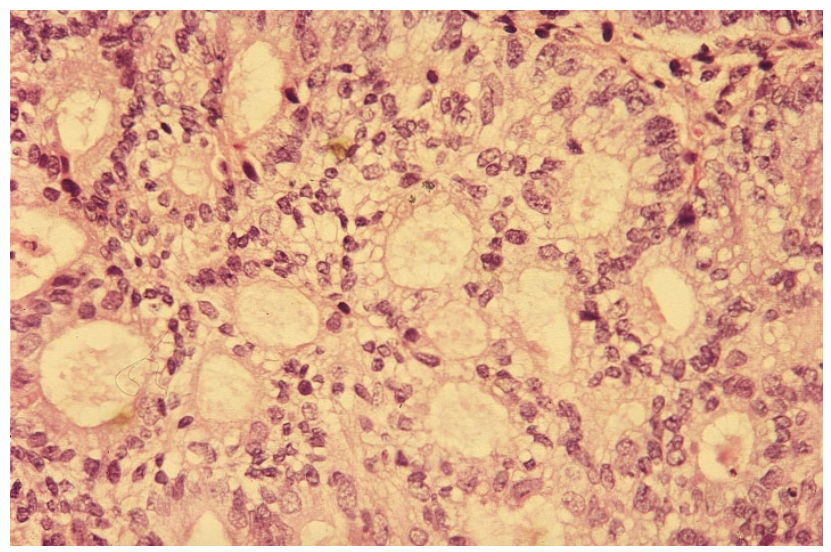

FIGURE 10. Same patient as in Figure 9, after 6 months more on high-potency progesterone (Megace). Endometrial biopsy shows cribriform pattern with secretory changes. Subsequent hysterectomy revealed an endometrial carcinoma invading $70 \%$ of myometrial thickness.

hysterectomy with extensive sampling of the endometrium can the absence of neoplasm be confirmed.

\section{Lupron therapy for uterine leiomyomas}

Gonadotropin releasing hormone agonists (Lupron) can effectively reduce the size of uterine leiomyomas in a majority of patients by inducing an iatrogenic reversible menopause caused by the suppression of estrogenic stimulation. The effect is a shrinkage of up to $70 \%$ or more of the volume of both leiomyomas and uteri, taking place over a relatively short period of time. This therapy generally does not exceed 3 months to minimize the side effects of an early menopause (30). The shrinking of the leiomyomatous nodules is due to an acceleration of a process that naturally takes place after menopause in which retraction with progressive hyalinization replaces the smooth muscle tumor, which is deprived of estrogenic stimulation. The histologic changes seen in the treated leiomyomas, usually removed by laparoscopic myomectomy, are edema and necrosis during the first weeks, followed by nodular hyalinization and occasional lymphoid cell infiltrates $(31,32)$. The endometrium involutes during this therapy. It appears weakly proliferative, inactive, and eventually atrophic.

\section{Tamoxifen therapy for breast neoplasms}

Tamoxifen is a nonsteroidal, synthetic triethylene estrogen derivative used successfully in the adjuvant therapy and prophylaxis of breast cancer. It binds to the estrogen receptors in a manner similar to that of estradiol and induces binding of the tamoxifen/receptor complex to the nuclear DNA, resulting in a decrease of available unbound receptors. This explains the antiestrogenic effect on breast tissue. Its effect on the uterus is more complicated, being both antagonistic and agonistic to that of estrogens (33-36). The estrogen-agonist effect on the uterus is manifested by polypoid endometrial proliferation with glandular hyperplasia ranging from simple to complex and atypical. The stroma of these polyps is usually fibrotic. The estrogen-agonist myometrial response to tamoxifen therapy may include diffuse smooth muscle hyperplasia (myohyperplasia), leiomyomata, and adenomyosis, with or without hyperplastic glandular changes. The size of the uterus can increase considerably, in some cases reaching more than $1200 \mathrm{~g}$ (Fig. 11). In a recent study on 277 endometrial samples from tamoxifen-treated patients, $20 \%$ showed estrogenic effects in their uterus (37). They consisted of endometrial polyps, glandular hyperplasia, and epithelial metaplasias, most often mucinous and squamoid (Fig. 12). Mucinous metaplasia of endometrial glands often mimics endocervical tissue, suggesting that the tissue obtained for biopsy did not include endometrium. Approximately $40 \%$ of biopsies taken from these patients showed inactive or atrophic changes, whereas in others, especially in the younger age groups, there were cyclic changes. Endometrial cancer was diagnosed in 10 cases, of which 5 were well-differentiated endometrioid carcinomas, confined to endometrial polyps, in a setting of endometrial hyperplasia. Less well-differentiated, nonendometrioid carcinomas, some invading the myometrium, were also seen in this material. High-grade endometrial tumors in patients receiving tamoxifen therapy have been reported in the literature $(38,39)$. When progesterone is added to the tamoxifen therapy, the endometrial carcinoma may exhibit an irregular and confusing pattern of secretory glands (Fig. 13). Endometrial carcinoma with secretory changes and deep myometrial invasion can also be seen with this therapy (Fig. 14) $(40,41)$. Carcinosarcomas (malignant mixed müllerian tumors) of the uterus have been reported as well, representing the second most

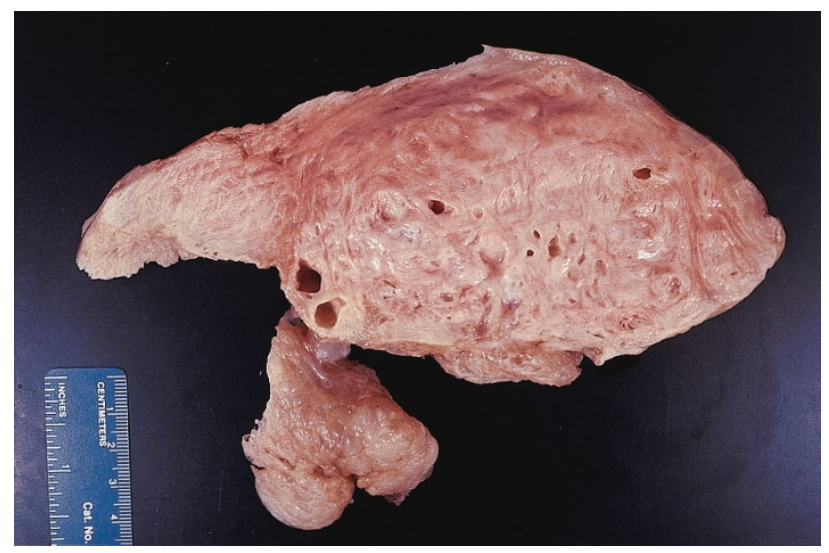

FIGURE 11. Tamoxifen therapy for breast cancer in a 76-year-old patient. Uterus weighed $1280 \mathrm{~g}$, showing diffuse adenomyosis, myohyperplasia, endometrial polyps, and atypical glandular hyperplasia. 


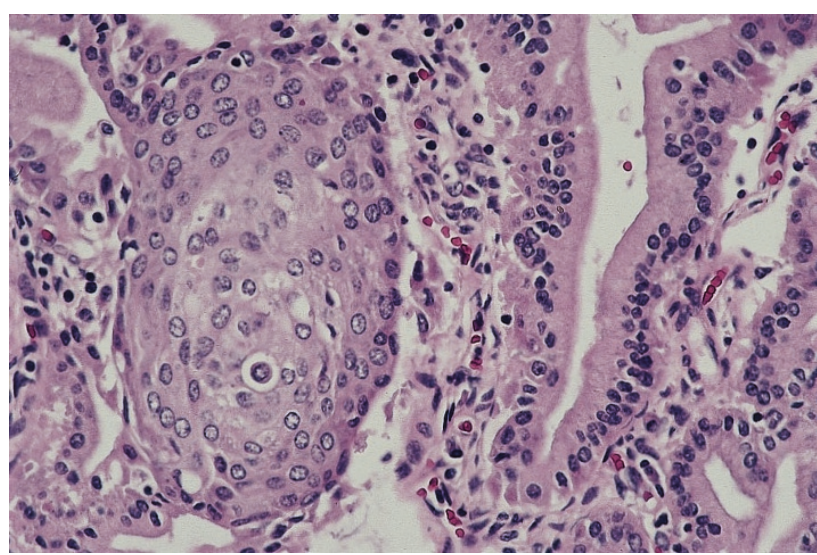

FIGURE 12. Tamoxifen therapy for breast cancer for 5 years. Endometrial biopsy with mucinous and squamous metaplasia, imitating endocervical tissue.

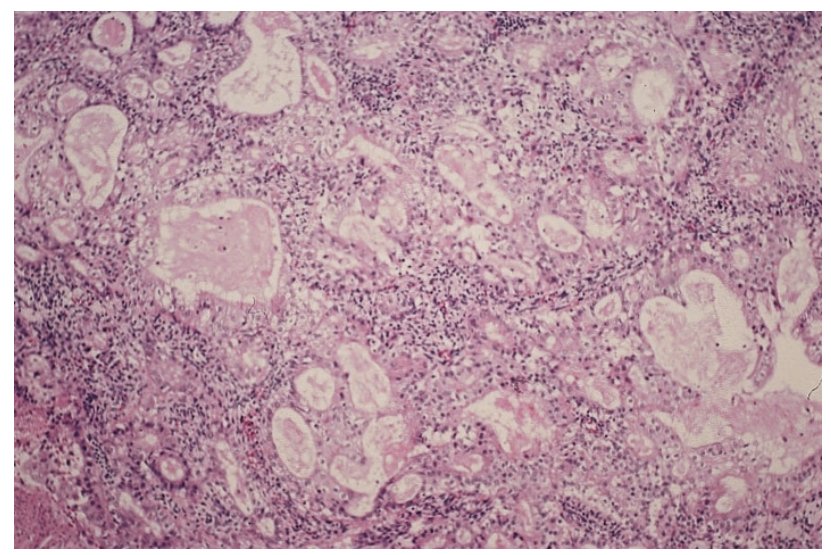

FIGURE 13. Tamoxifen therapy for breast cancer for 8 years and medroxyprogesterone acetate therapy added for the past 2 years. Endometrial adenocarcinoma with irregular glandular secretory changes.

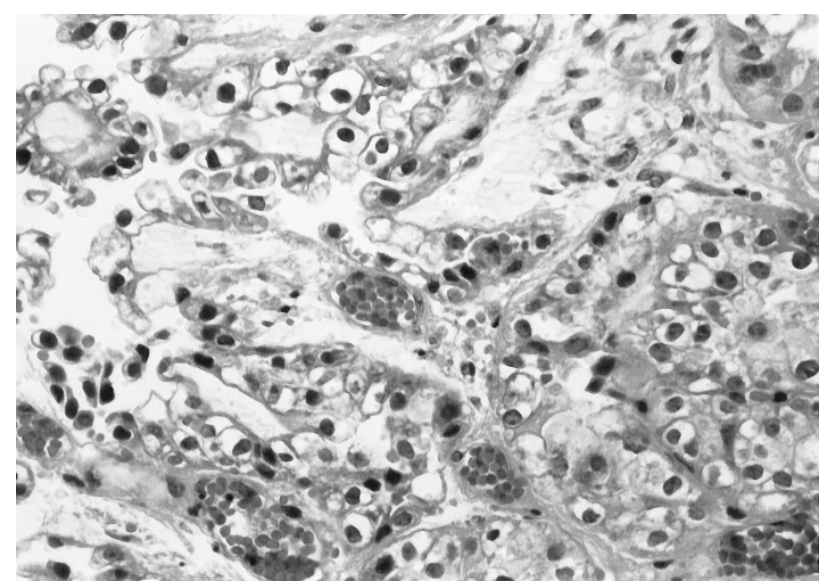

FIGURE 14. Tamoxifen therapy for breast cancer for 10 years and progesterone therapy (Megace) added for the past 4 years. Endometrial adenocarcinoma with secretory vacuoles seen on biopsy. The tumor invaded $70 \%$ of the myometrial thickness at subsequent hysterectomy.

common malignancy to be associated with tamoxifen therapy, as have adenosarcomas (Table 4) $(42,43)$.

The causal relationship between tamoxifen therapy for breast cancer and endometrial cancer is
TABLE 4. Tamoxifen for Breast Cancer: Changes in Uterus

Weakly proliferative, inactive, atrophic endometrium

Functional (proliferative, secretory) endometrium

Metaplasia: mucinous, papillary, other

Estrogen effects

Leiomyoma, adenomyosis, myohyperplasia

Endometrial polyps, without or with hyperplasia: simple cystic glandular, complex, atypical hyperplasia

Malignant changes

Endometrial carcinoma: endometrioid, nonendometrioid malignant mixed müllerian tumors (rare)

difficult to assess. Both breast and endometrial cancers are relatively common malignancies that affect a female population with similar risk factors: nulliparity, hyperestrogenism, and obesity, as well as the recently established hereditary genetic mutations. Endometrial cancer occurs in patients who have been diagnosed with breast cancer and who are not treated with tamoxifen (34). It often occurs in the female population at risk because of hyperestrogenism. Most of these cases are characterized as type 1 endometrial carcinoma with coexisting endometrial hyperplasia (44). Type 2 endometrial carcinoma, characterized by a more aggressive biologic behavior, is seen more often in elderly patients and is histologically less differentiated, more often nonendometrioid, and not associated with endometrial hyperplasia (44-46). Whereas type 1 is usually seen in hyperestrogenic patients, type 2, in most cases, probably develops without estrogen mediation. That type 2 endometrial carcinomas occur in tamoxifen-treated patients suggests an independent carcinogenic process, whereas the type 1 endometrial carcinoma in tamoxifen-treated patients may or may not be causally related to the therapy (47).

The benefits obtained from the effective and widely prescribed tamoxifen therapy for breast cancer exceed the possible risk of uterine neoplasm by far. However, screening for and prevention of this undesirable side effect of tamoxifen is indicated, both with sonographic imaging of the uterus and periodic endometrial biopsies.

Acknowledgment: The author thanks Ms. Mildred J. Levenson for secretarial assistance.

\section{REFERENCES}

1. Noyes RW. Normal phases of the endometrium. In: Norris JH, Hertig AT, Abell MR, editors. The uterus. Baltimore: Williams \& Wilkins; 1973. pp. 110-35.

2. Ferenczy A. Anatomy and histology of the uterine corpus. In: Kurman RJ, editor. Blaustein's pathology of the female genital tract. 4th ed. New York: Springer Verlag; 1994. pp. 332-52.

3. Soules MR, Wiebe RH, Aksel S, Hammond CB. The diagnosis and therapy of luteal phase deficiency. Fertil Steril 1977;18:1033-7. 
4. Deligdisch L, Yedwab G, Persitz A, David MP. Ultrastructural features in normal and hyperplastic postmenopausal endometrium. Acta Obstet Gynecol 1978;57:4439-52.

5. Gurpide E, Tseng L, Gusberg SB. Estrogen metabolism in normal and neoplastic endometrium. Am J Obstet Gynecol 1977;129:809-16.

6. Kelley HW, Miles PA, Buster JE, Scragg WH. Adenocarcinoma of the endometrium in women taking sequentially oral contraceptives. Obstet Gynecol 1976;47:200-2.

7. Silverberg SG, Makowski EL. Endometrial carcinoma in young women taking oral contraceptive agents. Obstet Gynecol 1975;46:503-6.

8. Cruz-Aquino M, Shenker L, Blaustein A. Pseudosarcoma of the endometrium. Obstet Gynecol 1967;29:93-6.

9. Ober WB. Effects of oral and intrauterine administration of contraceptives on the uterus. Hum Pathol 1977;8:513-27.

10. Navot D, Anderson TL, Droesch K. Hormonal manipulation of endometrial maturation. J Clin Endocrinol Metab 1989;68:801-7.

11. Thatcher SS, Donachie KM, Glasier A, Hillier SG, Baird DT. The effects of clomiphene citrate on the histology of human endometrium in regularly cycling women undergoing in vitro fertilization. Fertil Steril 1988;49:296-301.

12. Garcia JE, Acosta AA, Hsiu J-G, Jones HW Jr. Advanced endometrial maturation after ovulation induction with human menopausal gonadotropin/human chorionic gonadotropin for in vitro fertilization. Fertil Steril 1984;41:31-5.

13. Sharma V, Whitehead M, Mason B, Pryse-Davies J, Ryder T, Dowsett $\mathrm{M}$, et al. Influence of superovulation on endometrial and embryonic development. Fertil Steril 1990;53:822-9.

14. Gusberg SB: Precursors of corpus carcinoma: estrogens and adenomatous hyperplasia. Am J Obstet Gynecol 1974;54:905-27.

15. Silverberg SG. Hyperplasia and carcinoma of the endometrium. Semin Diagn Pathol 1988;5:135-53.

16. Brinton LA, Hoover RN. The endometrial cancer collaborative group. Estrogen replacement therapy and cancer risk: unresolved issues. J Obstet Gynecol 1993;81:165-71.

17. Prough SG, Aksel S, Wiebe RH, Shepherd J. Continuous estrogen/progestin therapy in menopause. Am J Obstet Gynecol 1987;157:1449-53.

18. Whitehead MI, Townsend PT, Pryse-Davis J, Ryder T, Lane G, Siddle N, et al. Effects of estrogens and progestogen on the biochemistry and morphology of the postmenopausal endometrium. Am J Obstet Gynecol 1982;142:791-5.

19. Voigt LF, Weiss NS, Chu J, Daling JR, McKnight B, van Belle G. Progestogen supplementation of exogenous estrogens and risk of endometrial cancer. Lancet 1991;338:274-7.

20. Whitehead MI, Frazer D. The effect of estrogens and progestogens on the endometrium. Obstet Gynecol Clin North Am 1987;14:299-320.

21. McGonigle KF, Karlan BY, Barbuto DA, Leuchter RS, Lagasse LD, Judd HL. Development of endometrial cancer in women on estrogen and progestin hormone replacement therapy. Gynecol Oncol 1994;55:126-32.

22. Jick SS. Combined estrogen and progesterone use and endometrial cancer. Epidemiology 1993;4:384 (letter to the editor).

23. Beresford SAA, Weiss NS, Voigt LF, McKnight B. Risk of endometrial cancer in relation to use of estrogen combined with cyclic progestagen therapy in post-menopausal women. Lancet 1997;349:458-61.

24. Vergote I, Kjorstad K, Abeler V, Kolstad P. A randomized trial of adjuvant progestagen in early endometrial cancer. Cancer 1989;64:1011-6.

25. Farhi DC, Nosanchuk J, Silverberg SG. Endometrial adenocarcinoma in women under 25 years of age. Obstet Gynecol 1986;68:741-5.

26. Randall TC, Kurman RJ. Progestin treatment of atypical hyperplasia and well differentiated carcinoma of the endometrium in women under age 40. Obstet Gynecol 1997;90:434-40.
27. Gerulath AH, Bonte R. Effect of progesterone on nucleic acid synthesis in vitro in carcinoma of the endometrium. Am J Obstet Gynecol 1977;28:722.

28. Deligdisch L, Holinka CF. Progesterone receptors in two groups of endometrial carcinoma. Cancer 1986;57:1385-8.

29. Holinka CK, Deligdisch L, Gurpide E. Histological evaluation of in vitro responses of endometrial adenocarcinoma to progestins and their relation to progesterone receptor levels. Cancer Res 1984;44:293-6.

30. Friedman AJ, Daly M, Juneau-Norcross M, Rein MS. Predictions of uterine volume reduction in women with myomas treated with a gonadotropin-releasing hormone agonist. Fertil Steril 1992;58:413-5.

31. Deligdisch L, Hirschmann S, Altchek A. Pathologic changes in gonadotropin releasing hormone agonist analogue treated uterine leiomyomata. Fertil Steril 1997;67:837-41.

32. Crow J, Gardner RL, McSweeney G, Shaw RW. Morphological changes in uterine leiomyomas treated by GnRH agonist Goserelin. Int J Gynecol Pathol 1995;14:235-42.

33. Seoud MA-F, Johns J, Weed JC Jr. Gynecologic tumors in tamoxifen-treated women with breast cancer. Obstet Gynecol 1993;82:165-9.

34. Cohen CJ. Tamoxifen and endometrial cancer: tamoxifen effects on the female genital tract. Semin Oncol 1997; 24(Suppl 1):S55-64.

35. Segna R, Dottino PR, Deligdisch L, Cohen CJ. Tamoxifen and endometrial cancer. Mt Sinai J Med 1992;59:416-8.

36. Satyaswaroop PG, Zaino RJ, Mortel R. Estrogen-like effect of tamoxifen on human endometrial carcinoma transplanted into nude mice. Cancer Res 1984;44:4006-10.

37. Deligdisch L, Cheng J, Saiz A. Endometrial changes in tamoxifen-treated patients. Abstract XIIth International Congress of the International Academy of Pathology, 1998, Nice, France.

38. Robinson DC, Bloss JD, Schiano MA. A retrospective study of tamoxifen and endometrial cancer in breast cancer patients. Gynecol Oncol 1995;59:186-90.

39. Magriples U, Naftolin F, Schwartz PE, Carcangiu ML. High grade endometrial carcinoma in tamoxifen-treated breast cancer patients. J Clin Oncol 1993;11:485-90.

40. Rendina GM, Donadio C, Fabri M, Mazzoni P, Nazzicone P. Tamoxifen and medroxyprogesterone therapy for advanced endometrial carcinoma. Eur J Obstet Gynecol Reprod Biol 1984;17:285-91.

41. Dallenbach-Hellweg G, Hahn U. Mucinous and clear cell adenocarcinomas of the endometrium in patients receiving anti-estrogens (tamoxifen) and gestagens. Int J Gynecol Pathol 1995;14:7-15.

42. Clement PB, Oliva E, Young RH. Mullerian adenosarcoma of the uterine corpus associated with tamoxifen therapy: a report of six cases and a review of tamoxifen-associated lesions. Int J Gynecol Pathol 1996;15:222-9.

43. Clarke MR. Uterine malignant mixed mullerian tumor in a patient on long-term tamoxifen therapy for breast cancer. Gynecol Oncol 1993;51:311-5.

44. Deligdisch L, Cohen CJ. Histologic correlates and virulence implications of endometrial carcinoma associated with adenomatous hyperplasia. Cancer 1985;56:1452-5.

45. Hofman K, Nekhliudov L, Deligdisch L. Endometrial carcinoma in elderly women. Gynecol Oncol 1995;58:198-201.

46. Deligdisch L, Kase NG, Bleiweiss IJ. Endometrial cancer in elderly women: a histologic and steroid receptor study. Gerontology 2000;46:17-21.

47. Schlesinger C, Kamoi S, Ascher S, Kendell M, Lage JM, Silverberg SG. Endometrial polyps: a comparison study of patients receiving tamoxifen with two control groups. Int J Gynecol Pathol 1998;17:302-11. 